\title{
Inside bad trips: Exploring extra-pharmacological factors
}

\author{
GENÍS ONA* \\ Department of Anthropology, Rovira i Virgili University, Tarragona, Spain
}

(Received: June 18, 2017; revised manuscript received: January 16, 2018; accepted: January 17, 2018)

\begin{abstract}
Objective: This study aimed to clarify the influence of extra-pharmacological factors in the etiology of bad trips, a common adverse reaction related to the consumption of psychedelic drugs. Methods: A descriptive approach was adopted. The information was collected using a web-based survey. The survey respondents volunteered to participate based on the condition that they had suffered a bad trip in the past. Results: This report reveals some variables that are commonly related to this adverse reaction (i.e., the recreational consumption of drugs, the consumption of drugs in large, open outdoor spaces, or being inexperienced with the drug). In addition, we note that some problems, which may be related to bad trips (i.e., mixing drugs, ignorance about the purity, or the dosage), can be solved through harmreduction strategies. Conclusions: We found certain aspects that could be related to the appearance of a bad trip, but it is not possible to establish a causal connection. We recommend conducting prospective studies with larger samples to collect more information about the role of extra-pharmacological factors.
\end{abstract}

Keywords: psychedelic, bad trip, drug, adverse reaction

\section{INTRODUCTION}

Psychedelic drugs like lysergic acid diethylamide (LSD-25), 3,4-methylenedioxymethamphetamine (MDMA), and psilocybin are being investigated again, showing therapeutic potential for certain psychological disorders, such as post-traumatic stress disorder (Amoroso \& Workman, 2016; Mithoefer et al., 2013; Oehen, Traver, Widmer, \& Schnyder, 2013) and depression and anxiety associated with life-threatening illnesses (Grob et al., 2011; McCorvy, Olsen, \& Roth, 2016). There are dozens of references on this issue, so we suggest reading the following recent reviews: Garcia-Romeu, Kersgaard, and Addy (2016) and Nichols, Johnson, and Nichols (2017).

Alongside this therapeutic trend, recent studies have begun to challenge the notion that the psychedelics are harmful to mental health (Krebs \& Johansen, 2013). On the contrary, we may consider the possibility that the consumption of these drugs by healthy people in naturalistic contexts could have benefits to mental health (Johansen \& Krebs, 2015; Ona \& Spuch, 2017).

We note that all drug users are exposed to some adverse reactions (Grof, 2005; Strassman, 1984). These substances can exacerbate the psychopathological traits that could cause persisting adverse reactions (Cohen, 1960; Johnson, Richards, \& Griffiths, 2008; Strassman, 1984). When drug users do not have previous psychopathological traits, they may experience acute adverse reactions like fear, panic, overwhelming anxiety, or confusion, which together constitutes what is commonly known as a bad trip. This phenomenon has not been studied in depth. While there are important number of studies, which address the adverse reactions related to the consumption of psychedelics (Abraham \& Aldridge, 1993; Bewley, 1967;
Carbonaro et al., 2016; Cohen \& Ditman, 1963; Fink, Simeon, Haque, \& Itil, 1966; Frosch, Robbins, \& Stern, 1965; Glickman \& Blumenfield, 1967; Heaton, 1975; Kleber, 1967; Malleson, 1971; Smart \& Bateman, 1967; Strassman, 1984), few studies specifically focus on bad trips (Barrett, Bradstreet, Leoutsakos, Johnson, \& Griffiths, 2016; Carbonaro et al., 2016; Cohen, 1960; Ungerleider, Fisher, Fuller, \& Caldwell, 1968).

Literature on psychedelic-induced states of consciousness suggests that the extra-pharmacological factors are determinant (Grof, 2005; Masters \& Houston, 1974; Prepeliczay, 2002; Sumnall, Cole, \& Jerome, 2006), because states of consciousness induced by psychedelics potentiate perception and experience of external stimuli (Grof, 2005).

The delineation of these extra-pharmacological factors is conceptualized in the notion of "set and setting," that is to say, the way the experience is influenced by the individual's mindset (set) as well as the surroundings and atmosphere (setting).

In this study, the relationship between bad trips and a delimited list of extra-pharmacological factors will be explored.

\section{METHODS}

\section{Overview}

An online survey was developed to collect information about bad trips. This was designed with the intention of discovering

* Corresponding address: Genís Ona; Interdisciplinary Psychedelic Studies, Medical Anthropology Research Center, Rovira i Virgili University, Avda. Catalunya, 35, Tarragona 43002, Spain; Phone: +34 675 553344; E-mail: genis.ona@e-campus.uab.cat

This is an open-access article distributed under the terms of the Creative Commons Attribution-NonCommercial 4.0 International License, which permits unrestricted use, distribution, and reproduction in any medium for non-commercial purposes, provided the original author and source are credited, a link to the CC License is provided, and changes - if any - are indicated. 
what, if any, extra-pharmacological factors are commonly related to the adverse reaction known as a bad trip.

\section{Materials}

The survey was designed with closed-ended questions with defined response categories. At the conclusion of the survey, participants were also given the opportunity to provide any other comments they wished to on the section topic.

The first section of the survey was used to determine participants' eligibility. Participants were provided with information about the survey, including the purpose and assurance of anonymity. Respondents were asked to recall their bad trip and answer the questions about that experience. If they had experienced more than one bad trip, they were asked to choose the one they considered to be the most significant.

The demographic section of the survey asked participants for information including age, education, occupation, and country of residence.

The main body of the survey had five sections concerning respondents' medical history, the context of the experience, the drug used, the experience, and the after-effects. A schematic diagram of the survey instrument is provided in Figure 1. The full survey is available from the authors upon request.

\section{Procedure}

The survey was hosted online using the Google Forms platform (docs.google.com/forms) and shared on social media with the collaboration of various organizations from Spain and other Latin American countries (e.g., Energy Control, Caapi, Échele Cabeza cuando se dé en la Cabeza, among others). Only drug users who had suffered a bad trip were requested to complete the survey.

\section{Sample}

A total of 233 volunteers aged between 18 and 54 years $(\bar{X}=23.6)$ completed the survey. Two subjects were excluded from the study, because the supposed bad trip they suffered did not emerge in response to the consumption of a psychedelic substance.

\section{Data analysis}

IBM SPSS Statistics v.21 was used to analyze the survey data. The results are reported in percentages.

\section{Ethics}

This study has been conducted in accordance with the ethical principles set out in the declaration of Helsinki.

\section{RESULTS}

\section{Sociodemographic information}

The majority of the sample reported had completed university studies $(64.5 \%)$, whereas $14.7 \%$ had completed highschool studies and $10.8 \%$ had master's or doctorate degrees.

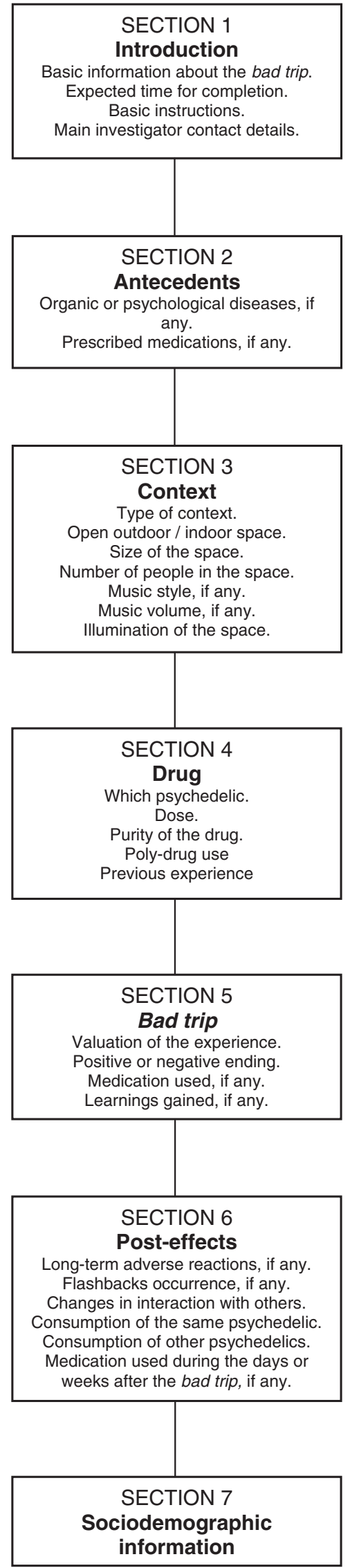

Figure 1. Schematic diagram of the survey 
About $48.7 \%$ of participants were students, whereas $46.1 \%$ were professionals and $5.2 \%$ were unemployed.

A majority of respondents reside in Colombia (40.7\%), whereas 11 other nationalities are represented in the results [Spain (28.5\%), Mexico (11.2\%), Argentina (9.1\%), Chile (3.4\%), Ecuador (1.3\%), Portugal (1.3\%), United States $(0.9 \%)$, France $(0.9 \%)$, Germany $(0.9 \%)$, Peru $(0.4 \%)$, and Panama $(0.4 \%)$; see this information in Table 1].

\section{Medical history of the sample}

About $78.3 \%$ of the sample did not report any physical condition before the bad trip. The rest of the sample reported asthma $(7.3 \%)$, hypoglycemia $(1.7 \%)$, gastritis $(1.3 \%)$, arrhythmia $(0.9 \%)$, atopic dermatitis $(0.9 \%)$, or hypothyroidism $(0.9 \%)$, among others.

Similarly, $76.6 \%$ of the sample did not have any psychological diagnosis. The remaining $22.4 \%$ reported anxiety $(6.9 \%)$, depression $(6.7 \%)$, attention-deficit hyperactivity disorder (ADHD) (1.7\%), bipolar disorder (1.7\%), schizophrenia $(0.8 \%)$, or sleep disorders $(0.4 \%)$.

Medications reported to have been consumed before the occurrence of the bad trip were ventolin $(2.1 \%)$, salbutamol $(1.7 \%)$, fluoxetine $(1.3 \%)$, quetiapine $(0.9 \%)$, loratadine $(0.9 \%)$, and clonazepam $(0.9 \%)$, among others. About $78.4 \%$ of the sample did not report any relevant medication (see this information in Table 1).

\section{Characteristics of the context}

Respondents were asked to classify the context of their bad trip as one of three different categories: therapeutic (looking for introspection), recreational (with a few friends), and festive (in raves or festivals). Most of the sample defined their context as recreational $(55.4 \%)$, with $30.3 \%$ as festive and $14.2 \%$ as therapeutic. As a curiosity, the percentage of respondents with psychological disorders were equally distributed between the three categories, $\chi^{2}(26, N=231)=$ $20.25, p=.77$.
The majority of the sample (57.1\%) took the drug in open outdoor spaces, whereas $42.4 \%$ took in indoor spaces.

Regarding the size of the space where the bad trip took place, $63.2 \%$ of the sample classified the space as large with $36.6 \%$ of them reporting that the size was "overwhelming." About $36.8 \%$ of the sample classified the space as small with $12.9 \%$ of them reporting that this size was "overwhelming."

There were less than 10 people in the same space in $44.1 \%$ of the cases; more than 10 in $27.7 \%$ of the cases; and more than 50 people in $13.4 \%$ of cases. About $14.7 \%$ of the sample reported having being alone during the time of the bad trip.

There was music playing in $78.4 \%$ of the cases. About $26.3 \%$ of respondents reported that they liked the music style, whereas $7.8 \%$ did not like it. We do not have information about the remaining $44.3 \%$. In this "music group," $37.6 \%$ indicated that music was at low volume, allowing for conversation; $23.8 \%$ that music was at high volume, hindering conversation; $11.2 \%$ that music was at very high volume, making any conversation impossible; and $5.6 \%$ that music was at very low volume.

Regarding the illumination of the context, $56.2 \%$ of respondents reported that the visibility was fairly good; $32 \%$ of the subjects reported the context was dark; $6.5 \%$ of the subjects reported there was too much light; and finally $5.2 \%$ of the subjects reported that the context was very dark (see this information in Table 2).

\section{Drug}

In $50.2 \%$ of the cases, the drug was LSD. Then, the drugs were smoked cannabis (14.7\%), psilocybin mushrooms (10.3\%), 25i-NBOMe (4.3\%), MDMA (3.9\%), Salvia divinorum $(3 \%)$, ingested cannabis $(2.2 \%)$, ayahuasca $(2.2 \%)$, magic mushrooms (psilocybin) (2.2\%), 2,5-dimethoxy-4bromophenethylamine $(2.2 \%)$, 4-ethyl-2,5-dimethoxyphenethylamine $(0.9 \%)$, ketamine $(0.9 \%)$, San Pedro (mescaline) $(0.9 \%)$, LSA $(0.4 \%), \mathrm{N}, \mathrm{N}$-dimethyltryptamine $(0.4 \%)$, and 5-methoxy-N-methyl-N-isopropyltryptamine $(0.4 \%)$.

Table 1. Sociodemographic information and medical history of respondents

\begin{tabular}{|c|c|c|c|c|c|}
\hline \multicolumn{6}{|c|}{ Sociodemographic information } \\
\hline \multirow[t]{2}{*}{ Age } & $<20$ & $20-30$ & $31-40$ & $41-50$ & $51-60$ \\
\hline & $52(23 \%)$ & $144(62.3 \%)$ & $28(12.1 \%)$ & $5(2.2 \%)$ & $1(0.4 \%)$ \\
\hline \multirow[t]{2}{*}{ Education level } & Primary & Secondary & Post-obligatory & University & Master or $\mathrm{PhD}$ \\
\hline & $2(0.9 \%)$ & $21(9.1 \%)$ & $34(14.7 \%)$ & $149(64.5 \%)$ & $25(10.8 \%)$ \\
\hline \multirow[t]{2}{*}{ Occupation } & Students & Professionals & Unemployed & & \\
\hline & $113(48.7 \%)$ & $106(46.1 \%)$ & $12(5.2 \%)$ & & \\
\hline \multirow[t]{2}{*}{ Country } & Colombia & Spain & Mexico & Argentina & Chile \\
\hline & $94(40.7 \%)$ & $66(28.5 \%)$ & $26(11.2 \%)$ & $21(9.1 \%)$ & $8(3.4 \%)$ \\
\hline \multicolumn{6}{|l|}{ Medical history } \\
\hline \multirow[t]{2}{*}{ Organic diseases } & Non-reported & Asthma & Hypoglycemia & Gastritis & Arrhythmia \\
\hline & $181(78.3 \%)$ & $17(7.3 \%)$ & $4(1.7 \%)$ & $3(1.3 \%)$ & $2(0.9 \%)$ \\
\hline \multirow[t]{2}{*}{ Psychological diseases } & Non-reported & Anxiety & Depression & ADHD & Bipolar disorder \\
\hline & $177(76.6 \%)$ & $16(6.9 \%)$ & $15(6.7 \%)$ & $4(1.7 \%)$ & $4(1.7 \%)$ \\
\hline \multirow[t]{2}{*}{ Prescribed medication } & Ventolin & Salbutamol & Fluoxetine & Quetiapine & Loratadine \\
\hline & $5(2.1 \%)$ & $4(1.7 \%)$ & $3(1.3 \%)$ & $2(0.9 \%)$ & $2(0.9 \%)$ \\
\hline
\end{tabular}

Note. ADHD: attention-deficit hyperactivity disorder. 
Table 2. Characteristics of the context and the drug used

\begin{tabular}{|c|c|c|c|c|c|}
\hline \multicolumn{6}{|l|}{ Context } \\
\hline \multirow[t]{2}{*}{ Type of context } & Recreational & Festive & Therapeutic & & \\
\hline & $128(55.4 \%)$ & $70(30.3 \%)$ & $33(14.2 \%)$ & & \\
\hline \multirow[t]{2}{*}{ Indoor/outdoor } & Open/outdoor & Indoor & & & \\
\hline & $132(57.1 \%)$ & $98(42.4 \%)$ & & & \\
\hline \multirow[t]{2}{*}{ Size } & Large & Small & & & \\
\hline & $146(63.2 \%)$ & $85(36.8 \%)$ & & & \\
\hline \multirow[t]{2}{*}{ No. of other people } & $<10$ & +10 & +50 & Alone & \\
\hline & $102(44.1 \%)$ & $64(27.7 \%)$ & $31(13.4 \%)$ & $34(14.71 \%)$ & \\
\hline \multirow[t]{2}{*}{ Music } & Music playing & Liked style & Low volume & High volume & \\
\hline & $181(78.4 \%)$ & $60(26.3 \%)$ & $87(37.6 \%)$ & $55(23.8 \%)$ & \\
\hline \multirow[t]{2}{*}{ Illumination } & Too much light & Good visibility & Dark & Very dark & \\
\hline & $15(6.5 \%)$ & $130(56.2 \%)$ & $74(32 \%)$ & $12(5.2 \%)$ & \\
\hline \multicolumn{6}{|l|}{ Drug } \\
\hline \multirow[t]{2}{*}{ Which drug } & LSD & Cannabis & Magic mushrooms & 25i-NBOMe & MDMA \\
\hline & $116(50.2 \%)$ & $34(14.7 \%)$ & $24(10.3 \%)$ & $10(4.3 \%)$ & $9(3.9 \%)$ \\
\hline \multirow[t]{2}{*}{ Dose } & Low & Medium & High & Not sure & \\
\hline & $15(6.5 \%)$ & $50(21.6 \%)$ & $25(10.8 \%)$ & $141(61.1 \%)$ & \\
\hline Purity & $\begin{array}{l}\text { Drug analyzed } \\
81(35 \%)\end{array}$ & $\begin{array}{c}\text { Not analyzed } \\
150(65 \%)\end{array}$ & & & \\
\hline \multirow[t]{2}{*}{ Other drugs } & Not mixed & Poly-drug use & & & \\
\hline & $76(32.8 \%)$ & $155(67.2 \%)$ & & & \\
\hline \multirow{2}{*}{ Previous experience } & First time & Little experience & Very experienced & & \\
\hline & $50(21.6 \%)$ & $112(48.4 \%)$ & $69(29.9 \%)$ & & \\
\hline
\end{tabular}

Note. LSD: lysergic acid diethylamide; MDMA: 3,4-methylenedioxymethamphetamine.

It is important to note that $60.8 \%$ of the sample did not know the dose (low, medium, or high) they took. Among those who knew the dose, $21.6 \%$ took a medium dose, $10.8 \%$ a high dose, and $6.5 \%$ a low dose.

Similarly, $65 \%$ of respondents were not aware of the purity of the drug. The remainder of the sample did objective analyses through harm-reduction organizations.

Notably, we found that $67.2 \%$ of our sample consumed more than one drug. The most common secondary drug was cannabis $(24.1 \%)$, followed by tobacco $(6 \%)$, alcohol $(6 \%)$, MDMA (0.4\%), speed (0.4\%), Brugmansia (0.4\%), and poppers $(0.4 \%)$ (see this information in Table 2$)$.

\section{Previous experience}

Respondents were asked about their previous experience with the drug. About $48.4 \%$ of the sample had little experience; $29.9 \%$ were very experienced; and $21.6 \%$ had never taken the drug.

\section{Valuation of the experience}

Subjects had to classify their experience using a Likert-type item with seven options, ranging from "it was the worse experience of my life" to "it was the best experience of my life." About $82.3 \%$ of subjects classified their experience negatively with $15.1 \%$ as the worst of their lives. About $17.7 \%$ of subjects classified their experience positively with $1.7 \%$ as the best experience of their lives.

It is important to know if subjects were able to redirect the bad trip or not. In our sample, $68.5 \%$ of subjects could in fact redirect their bad trip. Moreover, $90.5 \%$ of the sample reported having had educational insights from the experience (e.g., not to mix different drugs, the importance of selecting an adequate context, not to take drugs, etc.).

\section{Use of medication}

This question refers to the use of medication to stop the bad trip. About $5.2 \%$ of respondents consumed medication with the intention of terminating the bad trip, and of these, the medications were diazepam (1.3\%), antipsychotic drugs (not specified) $(0.9 \%)$, bromazepam $(0.4 \%)$, caffeine $(0.4 \%)$, or cocaine $(0.4 \%)$.

\section{After-effects}

About $25.4 \%$ of subjects reported long-term side effects, defined as symptoms or adverse reactions sustained for weeks or months. These included anxiety, depression, and dissociative symptoms.

About $44.8 \%$ of subjects experienced some flashback, which consisted of reexperiencing the perceptual effects induced by psychedelic drugs at a later time.

Despite this adverse reaction, $64.2 \%$ of respondents reported having taken the same drug again. About $65.1 \%$ consumed the same drug along with other substances.

Regarding medication used days or weeks after the bad trip , $6.5 \%$ of subjects used anxiolytics, $12 \%$ antipsychotics, and $20 \%$ other medications like aspirin or ibuprofen.

\section{DISCUSSION}

The aim of this study was to examine the influence of extrapharmacological factors in the emergence of the bad trip. 
Our sample was very heterogeneous, coming from different countries and with unevenly aged strata. Because of this, we should carefully interpret the findings.

As far as we know, personality is a factor frequently associated with the development of a bad trip (Barrett, Johnson, \& Griffiths, 2017; Grof, 2005; Lienert \& Netter, 1996). The personality structure of our respondents was not assessed, but we observed that $22.4 \%$ of our sample had a psychiatric diagnosis prior to the occurrence of the bad trip. This information is not enough to assert that people with mental disorders are at higher risk of suffering a bad trip, but evidence supports the idea that this population is more susceptible (Cohen, 1960; Frosch et al., 1965; Robbins, Frosch, \& Stern, 1967; Strassman, 1984). We can ask ourselves about possible differences in the prevalence of mental disorders between our sample and the general population. In that case, we will have to face the difficulty of comparing the percentages observed in this study with the general prevalence in different countries, which could be different. In Colombia or Spain, the percentage of the population with psychiatric diagnoses is similar (around 9\%; Encuesta Nacional de Salud Mental [ENSM], 2015; Instituto Nacional de Estadística [INE], 2014). This value is well below that observed in our sample. For countries like Mexico, this information could not be found. It would also be interesting to compare the percentage of psychedelic consumers with psychiatric diagnoses who never developed a bad trip to the percentage of consumers who did. This is a controversial debate, which is thoroughly exemplified in Johansen and Krebs's (2015) study and the subsequent replies (Nesvag, Bramness, \& Ystrom, 2015).

The majority of the sample $(85.7 \%)$ came from recreational and festival contexts, so we can suggest that consumers who take psychedelic drugs in therapeutic contexts are less likely to suffer a bad trip, but it is not possible to conclude that the consumption of psychedelics in therapeutic contexts is favorable. We suggest considering the hypothesis that different contexts are chosen by different people, which implies a difficulty in associating certain contexts with concrete experiences. In addition, it is possible that people who choose to take psychedelic substances in therapeutic contexts are more aware of the so-called "set and setting," deliberately avoiding excessive noise, crowds, and other potentially adverse stimuli.

An indoor context seems to be more favorable to an open outdoor setting. The occurrence of a bad trip was mildly higher in bigger settings. However, $44 \%$ of individuals surveyed were with fewer than 10 people in the setting, which might seem contradictory.

About $30.6 \%$ of the respondents reported that there was no music in the context. We can find recommendations of using music in the literature related to the therapeutic use of psychedelics (Bonny \& Pahnke, 1972; Gaston \& Eagle, 1970; Grof, 2005; Johnson et al., 2008), but we cannot extrapolate this information to the illicit use. Recently, Kaelen et al. (2015) found that one's emotional response to music is enhanced by LSD, but that both positive and negative moods can be facilitated so consumers must be cautious, especially if the music is not appropriately selected. An assumption about the role of music could be that it will facilitate more bad trips when people do not like the style, but in this case, we found the contrary: $26.3 \%$ reported that they liked the style, whereas $7.8 \%$ did not like it. With respect to the volume, we found more frequency of bad trips when the music was at high or low volume $(37.9 \%$ and $23.7 \%$, respectively), whereas contexts with music at very high or very low volumes seem more "protective." Regarding that issue, Preller et al. (2017) reported that LSD could turn senseless music into beautiful music, suggesting that this factor might not be determinant for bad trips. Music is a complex variable that must be studied in future research.

Brightly lit spaces produced more bad trips (56.6\%), but dark spaces are also inadequate $(31.9 \%)$. It seems that the best option is a soft, warm light that allows one to see properly/for clear vision.

LSD was the most reported drug (50\%), but it is problematic to state that LSD causes bad trips more frequently. First, because the possibility exists that many more people in general take LSD than other drugs. In fact, a study by Carvalho et al. (2014) produced very similar percentages (53\% of the sample took LSD). Second, we are mainly referring to the use of multiple drugs simultaneously: $67.2 \%$ of the sample used more than one drug and $45 \%$ of the LSD users used other drugs.

With respect to the dose, medium doses were responsible for more bad trips (21.6\%), followed by high doses $(11.2 \%)$. Based on evidence gathered from this survey, it may be advisable to start with low doses until getting more experience, as $48 \%$ of respondents had little prior experience with the drug.

Knowledge of dose and purity of the drug consumed is a critical aspect in shaping the outcome of the trip. About $60 \%$ of the sample did not know the dose that they took and $64 \%$ did not have information about the purity of the drug. Drug users need to have this information to predict and manage the effects and prevent most common complications.

The findings about previous experience with the drug are quite interesting. We know that both experienced and naive users are at risk of suffering an acute adverse reaction (Robbins et al., 1967). However, the bigger percentage of bad trips happened when the subject had little experience. We may propose the following hypothesis: new consumers have more respect or fear in the face of a new drug, so they are more cautious. On the other side, subjects with plenty of experience with the drug could be less prone to develop adverse reactions, because they precisely know the common effects. But consumers with little experience can commit the error of taking too big of a dose or not having enough respect for the drug that they think they are familiar with.

About $17.7 \%$ of the sample classified the bad trip experience positively. We can understand this counterintuitive finding, if we consider the clinical observations of psychedelic therapists. They have reported that the resolution of psychologically challenging experiences may result in attribution of meaning, spiritual significance, and increased life satisfaction (Richards, 2015). Carbonaro et al. (2016) found that same result in a bigger percentage (84\%) of their sample.

About $5.2 \%$ of respondents consumed medication with the intention of terminating the bad experience. Among those, we found benzodiazepines (1.7\%), antipsychotic drugs $(0.9 \%)$, caffeine $(0.4 \%)$, or cocaine. The use of psychiatric medication should be considered only in cases in which the psychological distress is insufficiently 
managed with reassurance (Johnson et al., 2008). In these cases, the pharmacological intervention of choice is benzodiazepines. Some antipsychotic drugs like haloperidol can exacerbate the psychosis-like effects of psychedelic drugs, so they should not be used as a rescue medication (Vollenweider, Vollenweider-Scherpenhuyzen, Bäbler, Vogel, \& Hell, 1998). The use of other drugs like caffeine or cocaine as a rescue medication is strongly discouraged not only because of the non-specific action on the ending of psychedelic effects, but also because of the possible aggravation of the anxiety state and the unknown purity of these drugs.

About $25.4 \%$ of our sample reported long-term side effects and $44.8 \%$ experienced flashbacks. If we conceive the bad trip as a really challenging and traumatic experience, the presence of long-term side effects is to be expected. In fact, this percentage is nearly the same as those found in other survey studies (Carbonaro et al., 2016). The percentage of flashbacks is much higher, reaching almost half of the sample. This is a very controversial phenomenon. Classic scientific literature suggests prevalence between $15 \%$ and $77 \%$ among consumers of psychedelic drugs (Blumenfield, 1971; Holsten, 1976; McGlothin \& Arnold, 1971; Shick \& Smith, 1970; Stanton \& Bardoni, 1972; Ungerleider et al., 1968). However, its existence has been recently questioned (Krebs \& Johansen, 2013).

\section{Limitations}

This study has important limitations that are crucial to consider. First, this study was made with an online selfadministered questionnaire. This kind of study assumes several complications: lack of understanding of some questions, exaggeration/minimization/omission of information, and dishonest answers, among others.

An effort was made to write very simple sentences, without any technical vocabulary. This has had a direct impact on the objectiveness or accuracy of the information. For example, in the question about the size of the context, subjective categories like "big/small" were used.

Participants from 12 different countries responded to our survey, amounting to a total of 233 subjects. We consider this to be a heterogeneous sample. In the interest of conducting a more accurate analysis, we would consider each country and their respective drug legislation, among other things. The prosecution and the stigma attached to drug use are very different in these countries. In most cases, this stigma could generate high levels of stress, which is sufficient to trigger or modulate adverse reactions.

Variables like personality or lifestyle could not be evaluated. They play a very important role in this kind of adverse reactions, so the present findings are subjected to a wide individual variability.

\section{CONCLUSIONS}

There are very few studies published on the bad trip phenomenon. This report offers a first step to increase the knowledge about this adverse reaction. Harm-reduction organizations may use this knowledge to provide more precise guidance and advice.
We found some aspects that could be related to the appearance of a bad trip, but it is not possible to establish a causal connection.

We suggest that future studies on the topic include prospective methodologies and consider variables like personality in the interest of producing more specific and reliable results.

Funding sources: No funding was obtained from public or private institutions.

Author's contribution: GO conceived and designed the survey, analyzed data, and wrote the paper.

\section{Conflict of interest: None.}

\section{REFERENCES}

Abraham, H. D., \& Aldridge, A. M. (1993). Adverse consequences of lysergic acid diethylamide. Addiction, 88(10), 1327-1334. doi:10.1111/j.1360-0443.1993.tb02018.x

Amoroso, T., \& Workman, M. (2016). Treating posttraumatic stress disorder with MDMA-assisted psychotherapy: A preliminary meta-analysis and comparison to prolonged exposure therapy. Journal of Pshychopharmacology, 30(7), 595-600. doi:10.1177/0269881116642542

Barrett, F. S., Bradstreet, M. P., Leoutsakos, J. S., Johnson, M. W., \& Griffiths, R. R. (2016). The challenging experience questionnaire: Characterization of challenging experiences with psilocybin mushrooms. Journal of Psychopharmacology, 30(12), 1279-1295. doi:10.1177/0269881116678781

Barrett, F. S., Johnson, M. W., \& Griffiths, R. L. (2017). Neuroticism is associated with challenging experiences with psilocybin mushrooms. Personality and Individual Differences, 117, 155-160. doi:10.1016/j.paid.2017.06.004

Bewley, T. H. (1967). Adverse reactions from the illicit use of lysergide. British Medical Journal, 3(5556), 28-30. doi:10.1136/bmj.3.5556.28

Blumenfield, M. (1971). Flashback phenomena in basic trainees who enter the U.S. Air Force. Military Medicine, 136(1), 39-41.

Bonny, H. L., \& Pahnke, W. N. (1972). The use of music in psychedelic (LSD) psychotherapy. Journal of Music Therapy, 9(2), 64-87. doi:10.1093/jmt/9.2.64

Carbonaro, T. M., Bradstreet, M. P., Barrett, F. S., MacLean, K. A., Jesse, R., Johnson, M. W., \& Griffiths, R. R. (2016). Survey study of challenging experiences after ingesting psilocybin mushrooms: Acute and enduring positive and negative consequences. Journal of Psychopharmacology, 30(12), 1268-1278. doi:10.1177/0269881116662634

Carvalho, M. C., de Sousa, M. P., Frango, P., Dias, P., Carvalho, J., Rodrigues, M., \& Rodrigues, T. (2014). Crisis intervention related to the use of psychoactive substances in recreational settings - Evaluating the Kosmicare Project at Boom Festival. Current Drug Abuse Reviews, 7(2), 81-100. doi:10.2174/ 1874473708666150107115515

Cohen, S. (1960). Historia del LSD [The beyond within: The LSD story]. Madrid, Spain: Edicusa. 
Cohen, S., \& Ditman, K. S. (1963). Prolonged adverse reactions to lysergic acid diethylamide. Archives of General Psychiatry, 8(5), 475-480. doi:10.1001/archpsyc.1963. 01720110051006

Encuesta Nacional de Salud Mental [ENSM]. (2015). Prevalencia de trastornos mentales en la población Colombiana [Prevalence of mental disorders in Colombia]. Retrieved from https://www.odc.gov.co/Portals/1/publicaciones/pdf/ consumo/estudios/nacionales/CO031102015-salud_mental_ tomoI.pdf

Fink, M., Simeon, J., Haque, W., \& Itil, T. (1966). Prolonged adverse reactions to LSD in psychotic subjects. Archives of General Psychiatry, 15(5), 450-454. doi:10.1001/archpsyc. 1966.01730170002002

Frosch, W. A., Robbins, E. S., \& Stern, M. (1965). Untoward reactions to lysergic acid diethylamide (LSD) resulting in hospitalization. The New England Journal of Medicine, 273(23), 1235-1239. doi:10.1056/NEJM196512022732302

Garcia-Romeu, A., Kersgaard, B., \& Addy, P. H. (2016). Clinical applications of hallucinogens: A review. Experimental and Clinical Psychopharmacology, 24(4), 229-268. doi:10.1037/ pha0000084

Gaston, E. T., \& Eagle, C. T. (1970). The function of music in LSD therapy for alcoholic patients. Journal of Music Therapy, 7(1), 3-19. doi:10.1093/jmt/7.1.3

Glickman, L., \& Blumenfield, M. (1967). Psychological determinants of "LSD reactions". The Journal of Nervous and Mental Disease, 145(1), 79-83. doi:10.1097/00005053-19670700000010

Grob, C. S., Danforth, A. L., Chopra, G. S., Hagerty, M., McKay, C. R., Halberstadt, A. L., \& Greer, G. R. (2011). Pilot study of psilocybin treatment for anxiety in patients with advancedstage cancer. Archives of General Psychiatry, 68(1), 71-78. doi:10.1001/archgenpsychiatry.2010.116

Grof, S. (2005). Psicoterapia con LSD [LSD psychotherapy]. Barcelona, Spain: La Liebre de marzo.

Heaton, R. K. (1975). Subject expectancy and environmental factors as determinants of psychedelic flashback experiences. The Journal of Nervous and Mental Disease, 161(3), 157-165. doi:10.1097/00005053-197509000-00002

Holsten, F. (1976). Flashbacks: A personal follow-up. Archiv für Psychiatrie und Nervenkrankheiten, 222(4), 293-304. doi:10.1007/BF00343238

Instituto Nacional de Estadística [INE]. (2014). Encuesta de morbilidad hospitalaria [Survey of hospital morbidity]. Retrieved from http://www.ine.es/prensa/np942.pdf

Johansen, P. O., \& Krebs, T. S. (2015). Psychedelics not linked to mental health problems or suicidal behavior: A population study. Journal of Psychopharmacology, 29(3), 270-279. doi:10.1177/0269881114568039

Johnson, M. W., Richards, W. A., \& Griffiths, R. R. (2008). Human hallucinogen research: Guidelines for safety. Journal of Psychopharmacology, 22(6), 603-620. doi:10.1177/0269 881108093587

Kaelen, M., Barrett, F. S., Roseman, L., Lorenz, R., Family, N., Bolstridge, M., Curran, H. V., Feilding, A., Nutt, D. J., \& Carhart-Harris, R. L. (2015). LSD enhances the emotional response to music. Psychopharmacology, 232(19), 3607-3614. doi:10.1007/s00213-015-4014-y

Kleber, H. D. (1967). Prolonged adverse reactions from unsupervised use of hallucinogenic drugs. Journal of Nervous and
Mental Disease, 144(4), 308-319. doi:10.1097/00005053196704000-00010

Krebs, T. S., \& Johansen, P. O. (2013). Psychedelics and mental health: A population study. PLoS One, 8(8), e63972. doi:10.1371/journal.pone.0063972

Lienert, G. A., \& Netter, P. (1996). LSD response in Eysenckian trait types identified by polypredictive CFA. Personality and Individual Differences, 21(6), 845-850. doi:10.1016/S01918869(96)00143-2

Malleson, N. (1971). Acute adverse reactions to LSD in clinical and experimental use in the United Kingdom. British Journal of Psychiatry, 118(543), 229-230. doi:10.1192/bjp.118.543.229

Masters, R. E. L., \& Houston, J. (1974). LSD. Los secretos de la experiencia psicodélica [Varieties of psychedelic experience: The classic guide to the effects of LSD on the human psyche]. Barcelona, Spain: Bruguera.

McCorvy, J. D., Olsen, R. H., \& Roth, B. L. (2016). Psilocybin for depression and anxiety associated with life-threatening illnesses. Journal of Psychopharmacology, 30(12), 12091210. doi: $10.1177 / 0269881116675771$

McGlothin, W., \& Arnold, D. (1971). LSD revisited. A ten-year follow-up of medical LSD use. Archives of General Psychiatry, 24(1), 35-49. doi:10.1001/archpsyc.1971.01750070037005

Mithoefer, M. C., Wagner, M. T., Mithoefer, A. T., Jerome, L., Martin, S. F., Yazar-Klosinski, B., Michel, Y., Brewerton, T. D., \& Doblin, R. (2013). Durability of improvement in posttraumatic stress disorder symptoms and absence of harmful effects or drug dependency after 3,4-methylenedioxymethamphetamine-assisted psychotherapy: A prospective long-term follow-up study. Journal of Psychopharmacology, 27(1), 28-39. doi:10.1177/0269881112456611

Nesvag, R., Bramness, J. G., \& Ystrom, E. (2015). The link between use of psychedelic drugs and mental health problems. Journal of Psychopharmacology, 29(9), 1035-1040. doi:10. 1177/0269881115596156

Nichols, D. E., Johnson, M. W., \& Nichols, C. D. (2017). Psychedelics as medicines: An emerging new paradigm. Clinical Pharmacology \& Therapeuticals, 101(2), 209-219. doi:10.1002/cpt.557

Oehen, P., Traver, R., Widmer, V., \& Schnyder, U. (2013). A randomized, controlled pilot study of MDMA $( \pm 3$, 4-methylenedioxymethamphetamine)-assisted psychotherapy for treatment of resistant, chronic post-traumatic stress disorder (PTSD). Journal of Psychopharmacology, 27(1), 40-52. doi:10.1177/0269881112464827

Ona, G., \& Spuch, J. (2017). What happens after your first trip? A longitudinal, prospective, pragmatic-oriented study. Journal of Transpersonal Research, 8(1), 41-54.

Preller, K. H., Herdener, M., Pokorny, T., Planzer, A., Kraehenmann, R., Stämpfli, P., Liechti, M. E., Seifritz, E., \& Vollenweider, F. X. (2017). The fabric of meaning and subjective effects in LSD-induced states depend on serotonin 2A receptor activation. Current Biology, 27(3), 451-457. doi:10. 1016/j.cub.2016.12.030

Prepeliczay, S. (2002). Socio-cultural and psychological aspects of contemporary LSD use in Germany. Journal of Drug Issues, 32(2), 431-458. doi:10.1177/002204260203 200207

Richards, W. A. (2015). Sacred knowledge: Psychedelics and religious experience. New York, NY: Columbia University Press. 
Robbins, E., Frosch, W. A., \& Stern, M. (1967). Further observations on untoward reactions to LSD. American Journal of Psychiatry, 124(3), 393-395. doi:10.1176/ajp.124.3.393

Shick, J. F. E., \& Smith, D. E. (1970). Analysis of the LSD flashback. Journal of Psychedelic Drugs, 3(1), 13-19. doi:10.1080/02791072.1970.10471357

Smart, R. G., \& Bateman, K. (1967). Unfavourable reactions to LSD: A review and analysis of the available case reports. Canadian Medical Association Journal, 97(20), 1214-1221.

Stanton, M., \& Bardoni, A. (1972). Drug flashbacks: Reported frequency in a military population. American Journal Psychiatry, 129(6), 751-755. doi:10.1176/ajp.129.6.751

Strassman, R. J. (1984). Adverse reactions to psychedelic drugs. A review of the literature. Journal of Nervous and Mental
Disease, 172(10), 577-595. doi:10.1097/00005053-19841000000001

Sumnall, H. R., Cole, J. C., \& Jerome, L. (2006). The varieties of ecstatic experience: An exploration of the subjective experiences of ecstasy. Journal of Psychopharmacology, 20(5), 670 682. doi:10.1177/0269881106060764

Ungerleider, J. T., Fisher, D. D., Fuller, M., \& Caldwell, A. (1968). The "bad trip" - The etiology of the adverse LSD reaction. American Journal of Psychiatry, 124(11), 1483-1490. doi:10.1176/ajp.124.11.1483

Vollenweider, F. X., Vollenweider-Scherpenhuyzen, M. F. I., Bäbler, A., Vogel, H., \& Hell, D. (1998). Psilocybin induces schizophrenia-like psychosis in humans via serotonin-2 agonist action. Neuroreport, 9(17), 3897-3902. doi:10.1097/ 00001756-199812010-00024 